\title{
Periodic ordering of clusters and stripes in a two-dimensional lattice model. II. Results of Monte Carlo simulation
}

\author{
N. G. Almarza, ${ }^{1}$ J. Pȩkalski, ${ }^{2}$ and A. Ciach $^{2}$ \\ ${ }^{1}$ Instituto de Química Física Rocasolano, \\ CSIC, Serrano 119, E-28006 Madrid, Spain \\ ${ }^{2}$ Institute of Physical Chemistry, Polish Academy of Sciences, 01-224 Warszawa, Poland
}

(Dated: July 2, 2021)

\begin{abstract}
The triangular lattice model with nearest-neighbor attraction and third-neighbor repulsion, introduced in [J. Pȩkalski, A. Ciach and N. G. Almarza, arXiv:1401.0801 [cond-mat.soft]] is studied by Monte Carlo simulation. Introduction of appropriate order parameters allowed us to construct a phase diagram, where different phases with patterns made of clusters, bubbles or stripes are thermodynamically stable. We observe, in particular, two distinct lamellar phases - the less ordered one with global orientational order and the more ordered one with both orientational and translational order. Our results concern spontaneous pattern formation on solid surfaces, fluid interfaces or membranes that is driven by competing interactions between adsorbing particles or molecules.
\end{abstract}




\section{INTRODUCTION}

Spontaneous pattern formation in two-dimensions (2d) can occur for length scales ranging from fractions of a nanometer to micrometers when ions, organic molecules, macromolecules, nanoparticles or colloid particles are adsorbed on solid substrates, interfaces between two fluid phases or on biological membranes [1-10]. In the case of membrane proteins, cluster formation enables biological functions that cannot be performed by single macromolecules. For this reason the self-assembly is very important for life processes. On the other hand, self-assembly of ions or nanoparticles on surfaces or interfaces can find applications in nanotechnology or in biomimetic systems.

The sum of all the effective interactions in such systems often has a form of the shortrange attraction and long-range repulsion potential (SALR) which was intensively studied

recently $[11-21]$. The repulsion is typically of electrostatic origin [22 25], or it is caused by polymeric brushes [26, 27], while the attraction results from the van der Waals and solventmediated interactions [3, 23-26, 28]. The size of the structural motifs depends on the ranges of the attractive and the repulsive parts of the SALR potential. In the case of charged nanoparticles or biologically relevant globular proteins in some solvents the thickness of the clusters or stripes is $\sim 2-3 \sigma$, where $\sigma$ is the particle diameter. A characteristic example is lysozyme in water [23, 29].

In order to study the pattern formation in such systems, we have introduced a $2 \mathrm{~d}$ triangular lattice model with attraction between the first neighbors and repulsion between the third neighbors [30]. In Ref.[30] the ground state (GS), the mean-field (MF) phase diagram and the correlation function in the Brazovskii approximation have been obtained. The ground state of the model consists of the vacuum and fully occupied lattice for small and for large values of the chemical potential $\mu$ respectively. In addition, for intermediate values of $\mu$ periodically ordered clusters, stripes or bubbles are present when the repulsion to attraction ratio $J^{*}$ is sufficiently large. We have also found that at the coexistence between the ordered phases of different symmetry the ground state is strongly degenerated, and the entropy per site does not vanish in the thermodynamic limit. The microscopic states present at the vacuum - ordered cluster phase coexistence consist of clusters that are located at distances larger than the repulsion range, but apart from this restriction the positions and the number of the clusters are arbitrary. We interpret the collection of the disordered microstates 
consisting of small noninteracting clusters as a cluster fluid (CF) phase. For weak repulsion, $J^{*}<1$, the clusters consist of 7 particles and have a hexagonal shape, whereas for $J^{*}>1$ four particles form a rhomboidal cluster. At the coexistence between the cluster and the stripe phases the ground state is also strongly degenerated, and for $J^{*}>1$ stripes of different lengths and rhomboidal clusters are packed as densely as possible under the constraint of no repulsion between different objects. In such microstates one direction is distinguished, and the rotational symmetry is broken. We call the collection of such microstates a "molten lamella" (ML).

The degeneracy of the ground state must influence the phase behavior for $T>0$ (with $T$ being the temperature). However, this effect cannot be properly described by the MF theories. The average density in the disordered phase is position-independent, and the contribution to the internal energy from the repulsive part of the potential is an increasing function of the density. For this reason in MF the periodically ordered cluster phase coexists for low $T$ with a disordered fluid of very low density, with the fraction of occupied sites $\rho \rightarrow 0$ for $T \rightarrow 0$. In the majority of the microstates of the CF phase, however, the distances between the clusters are larger than the range of the repulsion. In addition, for $J^{*}>1$ the clusters are small and no intra-cluster repulsion is present, therefore the repulsion contribution vanishes even for large volume fractions. Thus, beyond MF the CF phase can be stable for quite large densities, $\rho \sim 0.2$, according to our simple estimation [30]. Similarly, the molten lamella phase is not present on the MF phase diagram for low $T$, although according to the GS analysis it is stable at the coexistence between the ordered cluster and stripe phases for $T=0$ [30].

On the other hand, for high $T$ thermal fluctuations destroy the periodic order. We have found an instability of the disordered phase with respect to density waves in MF. However, when fluctuations are taken into account in the Brazovskii-like approximation [31], the instability with respect to the density waves is shifted to $T=0$ [30]. On the other hand, a continuous transition between the isotropic phase and the phase with broken rotational symmetry was predicted recently for the Brazovskii functional at two-loop level [32].

The above observations indicate that in the SALR systems the role of mesoscopic fluctuations is crucial for the stability of different phases consisting of aperiodic distribution of some structural motifs.

In this work we are interested in effects of fluctuations on pattern formation in thermal 
equilibrium. We focus on the case of strong repulsion and perform Monte Carlo (MC) simulation on the triangular lattice model for $J^{*}=3$. Our main goal is the construction of the phase diagram. We should stress that because of difficulties in the simulation procedure and in the interpretation of the results only sketches of a phase diagram for the SALR potential have been obtained in 2d by $\mathrm{MC}$ [15] and in 3d by molecular dynamics [33].

In the next section the model and the GS are described briefly. The MC simulations are described in sec. III. The last section contains comparison between the MF and MC results, further discussion and summary.

\section{THE MODEL AND ITS GROUND STATE}

\section{A. The model}

We assume that the particles can occupy sites of a triangular lattice with the lattice constant equal to the diameter of the particles $\sigma$. The lattice sites are $\mathbf{x}=x_{1} \mathbf{e}_{1}+x_{2} \mathbf{e}_{2}$, where $\mathbf{e}_{1}, \mathbf{e}_{2}$ and $\mathbf{e}_{3}=\mathbf{e}_{2}-\mathbf{e}_{1}$ are the unit lattice vectors on the triangular lattice, i.e. $\left|\mathbf{e}_{1}\right|=\left|\mathbf{e}_{2}\right|=\left|\mathbf{e}_{1}-\mathbf{e}_{2}\right|=1$ (in $\sigma$-units), and $x_{i}$ are integer, $0 \leq x_{i} \leq L-1$, where $L$ is the size

of the lattice in the directions $\mathbf{e}_{1}$ and $\mathbf{e}_{2}$. We assume periodic boundary conditions (PBC), $L \equiv 0$ and $-1 \equiv L-1$. The number of sites on the lattices will be $M=L^{2}$.

The probability of a particular microscopic state $\{\hat{\rho}(\mathbf{x})\}$, where $\hat{\rho}(\mathbf{x})=1(0)$ when the site $\mathbf{x}$ is (is not) occupied, has the form

$$
p[\{\hat{\rho}(\mathbf{x})\}]=\Xi^{-1} \exp (-\beta H[\{\hat{\rho}(\mathbf{x})\}])
$$

where $\beta=1 /\left(k_{B} T\right), k_{B}$ is the Boltzmann constant,

$$
\Xi=\sum_{\{\hat{\rho}(\mathbf{x})\}} \exp (-\beta H[\{\hat{\rho}(\mathbf{x})\}])
$$

and the Hamiltonian is given by

$$
H=\frac{1}{2} \sum_{\mathbf{x}} \sum_{\mathbf{x}^{\prime}} \hat{\rho}(\mathbf{x}) V\left(\mathbf{x}-\mathbf{x}^{\prime}\right) \hat{\rho}\left(\mathbf{x}^{\prime}\right)-\sum_{\mathbf{x}} \mu \hat{\rho}(\mathbf{x}),
$$

with the interaction energy between the cells at $\mathbf{x}$ and $\mathbf{x}+\Delta \mathbf{x}$ 


$$
V(\Delta \mathbf{x})= \begin{cases}-J_{1} & \text { for }|\Delta \mathbf{x}|=1, \\ +J_{2} & \text { for }|\Delta \mathbf{x}|=2, \\ 0 & \text { otherwise. }\end{cases}
$$

A similar model, but on a square lattice and with next-nearest-neighbor repulsion was studied in Ref.[34]. The advantage of the triangular lattice is the possibility of close packing of the particles. Following Ref.[30, 35], we choose $J_{1}$ as the energy unit, and introduce the notation $X^{*}=X / J_{1}$ for any quantity $X$ with dimension of energy.

As shown in Ref.[30, 35], the phase diagram is symmetric with respect to the symmetry axis $\mu^{*}=\tilde{V}^{*}(0) / 2=3\left(J^{*}-1\right)$, with $J^{*}=J_{2} / J_{1}$. Because of the model symmetry, we consider only $\mu^{*} \leq \tilde{V}^{*}(0) / 2$ and take into account that the sites which for $\mu^{*}$ are occupied (empty), must be replaced by the empty (occupied) sites for $\mu^{*}=\tilde{V}^{*}(0)-\mu^{*}$.

\section{B. The ground state}

The $\left(J^{*}, \mu^{*}\right)$ GS has been determined in Ref.[30, 36]. Here we limit ourselves to strong repulsion, and choose $J^{*}=3$. The phase diagram and structure of the stable phases for $J^{*}=3$ at $T^{*}=0$ are shown in Fig, 1.

In order to discuss the GS, let us first consider the energy. For strong repulsion the energy assumes a minimum when neither intra-cluster nor inter-cluster repulsion is present, and as many nearest-neighbors as possible are occupied. The largest cluster with no intra-cluster repulsion has the form of a rhombus. The energy assumes a minimum for the largest number of noninteracting rhombuses per unit area, as shown in Fig.1b.

The energy competes with $-\mu N$, where $N$ is the number of occupied sites, and for $\mu \ll 0$ the minimum of $H^{*}[\{\hat{\rho}(\mathbf{x})\}] / L^{2}$ corresponds to the empty lattice (see Eq.(3)) . The vacuum and the ordered cluster phases coexist for $\mu^{*}=-5 / 4$. For $\mu^{*}=-5 / 4$ the disordered CF phase with a typical state shown in Fig $1 \mathrm{~g}$ c coexists with the other two phases stable for $\mu^{*}=-5 / 4$. For $-5 / 4 \leq \mu^{*} \leq 11 / 2$ the ordered rhombus (OR) phase is stable. Large $\mu$ can overcompensate the effect of the repulsion, and for $\mu^{*} \geq 11 / 2$ the lamellar (stripe) phase is stable. For $\mu^{*} \geq 6$ the inverse phases are stable for $\mu^{*}$ determined by the symmetry of the model (see Fig.1). The OR and the lamellar (L) phases coexist for $\mu^{*}=11 / 2$. At the coexistence between the $\mathrm{OR}$ and the $\mathrm{L}$ phases there exists a large number of disordered 


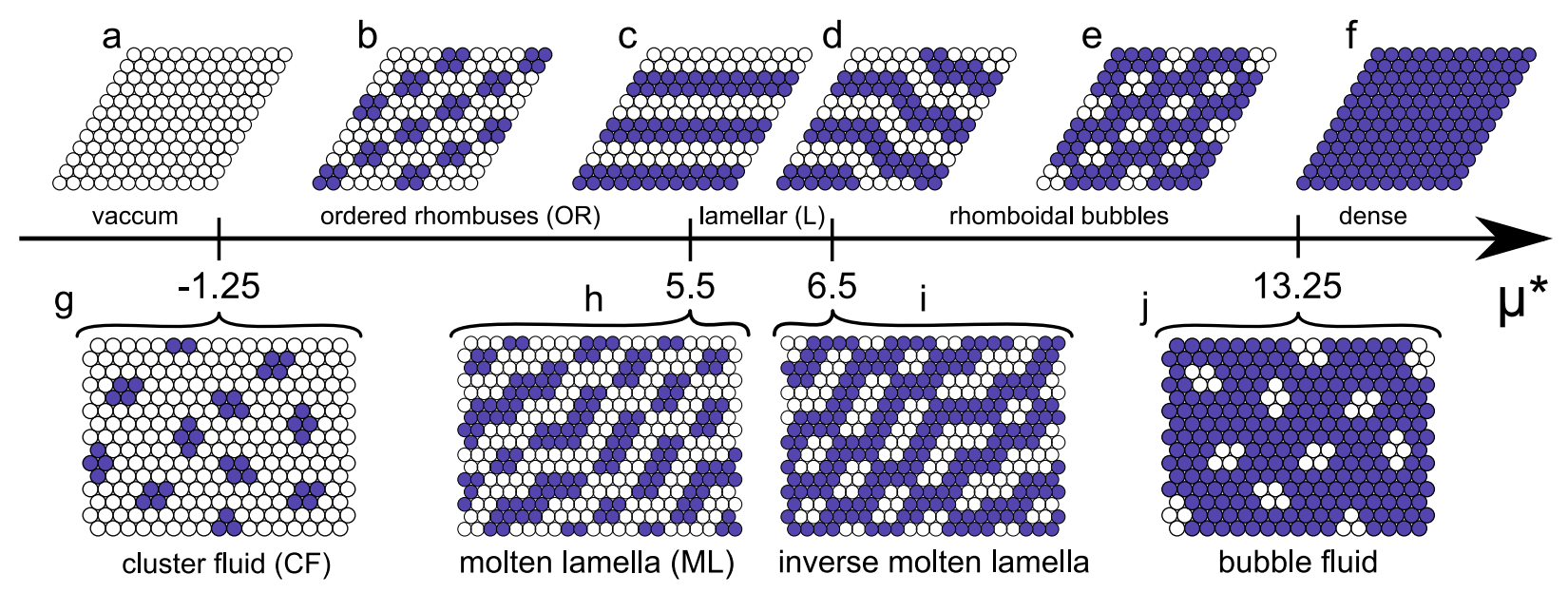

FIG. 1: Ground state of the model for $J^{*}=3$. The structures of the ordered phases are shown in the panels a-j, with: a) vacuum stable for $\mu^{*} \leq-5 / 4$, b) ordered rhomboidal phase (OR) stable for $-5 / 4 \leq \mu^{*} \leq 11 / 2$, c) and d) lamellar phase (L) stable for $11 / 2 \leq \mu^{*} \leq 13 / 2$, e) rhomboidal bubbles stable for $\left.13 / 2 \leq \mu^{*} \leq 53 / 4, \mathrm{f}\right)$ dense phase stable for $\mu^{*} \geq 53 / 4$. In the second row representative examples of the microscopic states of the disordered phases stable at the coexistences between the ordered phases are presented; g) the cluster fluid phase $(\mathrm{CF})$ stable for $\left.\mu^{*}=-5 / 4, \mathrm{~h}\right)$ molten lamella phase (ML) stable for $\left.\mu^{*}=11 / 2, \mathrm{i}\right)$ the inverse molten lamella stable for $\mu^{*}=13 / 2$ and $\mathrm{j}$ ) the bubble fluid phase stable for $\mu^{*}=53 / 4$.

states with the same value of the Hamiltonian as for the two coexisting ordered phases. A characteristic example of such states is shown in Fig.1 h. Such a structure is characterized by the lack of periodicity in all three lattice directions, but one direction ( $\hat{\mathbf{e}}_{3}$ in Fig $1 \mathrm{~h}$ ) is distinguished. We call the nonisotropic phase with noninteracting pieces of the zig-zag lamella of different length a 'molten lamella'.

Note that the Hamiltonian takes the same value in the lamellar phase shown in Fig 11; and in the zig-zag lamellar phase shown in Fig:1d. In what follows we will describe how the GS lamellar structures can be constructed and characterized. This description will allow us to make an estimation of the entropy of this phase, which will be useful later to find the phase transitions. Let us consider lamellas with periodicity in direction $\mathbf{e}_{3}$, as shown in Fig. 2. We start by setting three occupied positions defining an elementary triangle on the left end of the lattice (labelled as 1 in Fig 21). In order to proceed, after setting this first triangle (or whatever triangle with an odd label) we occupy a lattice position that closes an adjacent triangle (chosen between those labelled with even numbers in Fig. 2, which are those at the 
top or at the right of the previous triangle). Then after each triangle with even index is added the next triangle has to be that on its right, since the choice of the one on the left would break the periodicity in direction $\mathbf{e}_{3}$.

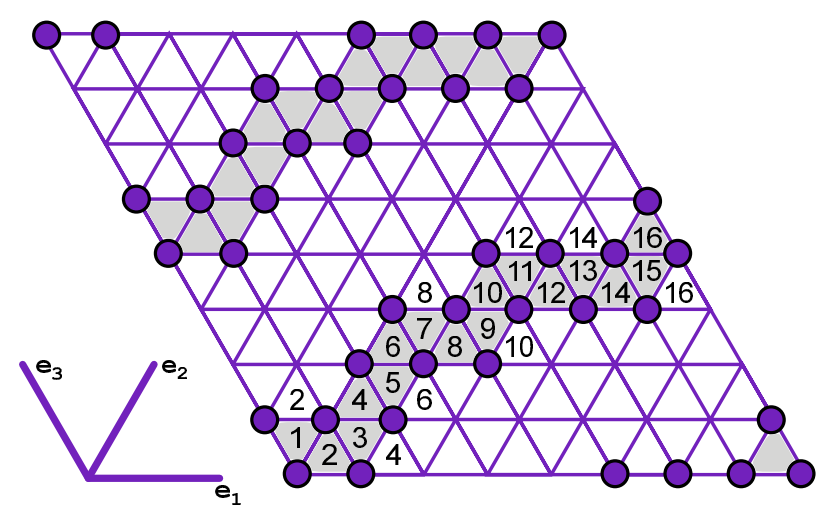

FIG. 2: Sketch of the construction of the ground state lamellar structures.

Once we get the reference lamellar stripe following the simple rules given above, it is straightforward to build up the GS lamellar structure by replicating each piece with translations. The occupied sites are $\mathbf{x}=\mathbf{x}_{0}+4 k \mathbf{e}_{3}$, where $\mathbf{x}_{0}$ is the position of the site at the reference stripe and $k>0$ is an integer number. The structures generated in this way fulfill $\rho=1 / 2$, and energy $U^{*} / N=-2+J^{*}$.

Taking into account the procedure to construct the GS lamellas it is possible to compute the entropy of this phase at $T^{*}=0$. PBC add some restrictions to the building procedure. If we follow one lamella stripe through $\mathrm{PBC}$, it is clear that the number of choices of growing the reference lamella stripe in the vertical direction must be zero or a multiple of four. In addition, $L$ must be a multiple of four.

Taking into account the number of ways of creating lamellar structures with translational order in, at least, one direction, and the restrictions due to $\mathrm{PBC}$, we can estimate the entropy of the system as

$$
S / L^{2}=\frac{k_{B}}{L^{2}} \ln Q \simeq \frac{k_{B} \ln 2}{L}+O\left(L^{-2}\right) .
$$

where $Q$ is the number of ways of building up the ordered lamellar structures following the previous procedure. The correction term $O\left(L^{-2}\right)$ includes the translational degeneracy from the location of the triangle 1 in Fig. 2, the possibility of considering three directions in the construction of the reference lamella stripe; the overcounting of straight lamellas, that show periodicity of length four in two directions; and the restrictions due to the PBC, that 
eliminate about $3 / 4$ of the reference stripes.

\section{MONTE CARLO SIMULATION}

Grand Canonical MC Simulations in rhombic boxes combined with thermodynamic integration techniques [37, 38] have been used to sketch the phase diagram of the model.

\section{A. Simulation methodology for finite temperatures}

Each MC move was carried out as follows: (1) A lattice position, $\mathbf{x}$, is chosen at random. Independently of the current state of this site, its new state for $\hat{\rho}(\mathbf{x})$ is chosen according to the possible interactions of a particle occupying the site $\mathbf{x}$. The ratio between the probabilities of choosing the two possible states is given as

$$
\frac{P(\hat{\rho}(\mathbf{x})=1)}{P(\hat{\rho}(\mathbf{x})=0)}=\exp \left\{\beta\left[\mu-\sum_{\mathbf{x}^{\prime}} V\left(\mathbf{x}-\mathbf{x}^{\prime}\right) \hat{\rho}\left(\mathbf{x}^{\prime}\right)\right]\right\} .
$$

According to the GS analysis we expect for $J^{*}=3$ the presence of two ordered phases at low temperature: lamellas with $\rho=1 / 2$ for values of $\mu^{*} \approx 6$, and the ordered rhombus phase for $-5 / 4<\mu^{*}<11 / 2$. At high temperature, the state of the sites should be essentially dictated by the value of the chemical potential, i.e., we will have a disordered phase with average density given as $\bar{\rho}=e^{\beta \mu} /\left(1+e^{\beta \mu}\right)$. Then we can expect some phase transition(s) connecting the high-temperature disordered phase with the low-temperature ordered phases. At low temperature and $\mu^{*} \approx-5 / 4$ we can expect to find the disordered $\mathrm{CF}$ phase composed of different aggregates of particles (mainly four particle clusters), while for increasing chemical potential the system should exhibit a phase transition into the OR phase. Similarly, for $\mu^{*} \approx 11 / 2$ the ML phase present in the GS is expected to be stable for $T>0$.

Taking above facts into account we have used thermodynamic integration (TI) techniques [37-39] to locate the phase transitions. These techniques are based on the numerical inte-

gration of thermodynamic potentials using simulation results. The integration of the grand potential is carried out as

$$
\Omega\left(L, \beta, \mu_{1}\right)=\Omega\left(L, \beta, \mu_{0}\right)-L^{2} \int_{\mu_{0}}^{\mu_{1}} \check{\rho}(L, \beta, \mu) \mathrm{d} \mu, \quad \text { where } \check{\rho} \equiv \frac{\langle N\rangle}{L^{2}},
$$


when the temperature is fixed, or:

$$
\Omega\left(L, \beta_{1}, \mu\right)=\Omega\left(L, \beta_{0}, \mu\right)+\int_{\beta_{0}}^{\beta_{1}} H(L, \beta, \mu) \mathrm{d} \beta,
$$

for fixed value of the chemical potential. The values of the grand potential in the thermodynamic limit are known for the ordered phases at low temperature, for the high-temperature limit $\beta=0$, and for the vacuum system $\rho=0$.

In the first attempt to compute the phase diagram we run sequences of simulations starting either from high temperatures $(\beta=0)$ or from low temperatures. In the latter case we used initial configurations of the corresponding stable phases at the GS. Then by applying TI we could get a draft of the shape of the phase diagram. We found some features in the results which introduced some difficulty in the analysis. When considering the sequences of simulations starting from high temperature, we found non-monotonic behavior with the system size for different properties. In addition to the expected transitions corresponding to the melting of the GS ordered structures, we found signatures of a number of additional thermodynamic transitions, which seemed to be either continuous or weakly first-order. Moreover, in some cases the number of these apparent transitions depends on the system size: As one increases the system size for a given value of $\mu$, the number of these transitions can increase. The presence of these transitions, and the atypical dependence of the results on the system size moved us to make use of parallel tempering (or replica exchange) MC sampling [40, 41] in order to improve the sampling quality of the simulation.

We have focused the simulation effort into a relatively large system, $L=120$, in order to get a reliable estimation of the phase diagram. Other system sizes were considered for some representative cases, and it was found that the topology of the phase diagram did not change and only minor displacements of the loci of the main transitions are to be expected. The phase diagram is presented in Fig. 3. In what follows we will address the different phase equilibria shown therein.

\section{B. Ordered rhombus and fluid phases}

\section{The limit of low temperature}

It is possible to compute the phase equilibria between the $\mathrm{OR}$ and $\mathrm{CF}$ phases in the limit $T \rightarrow 0$ (for $\mu^{*}=-5 / 4$ ). In this limit the system is composed exclusively by noninteracting 

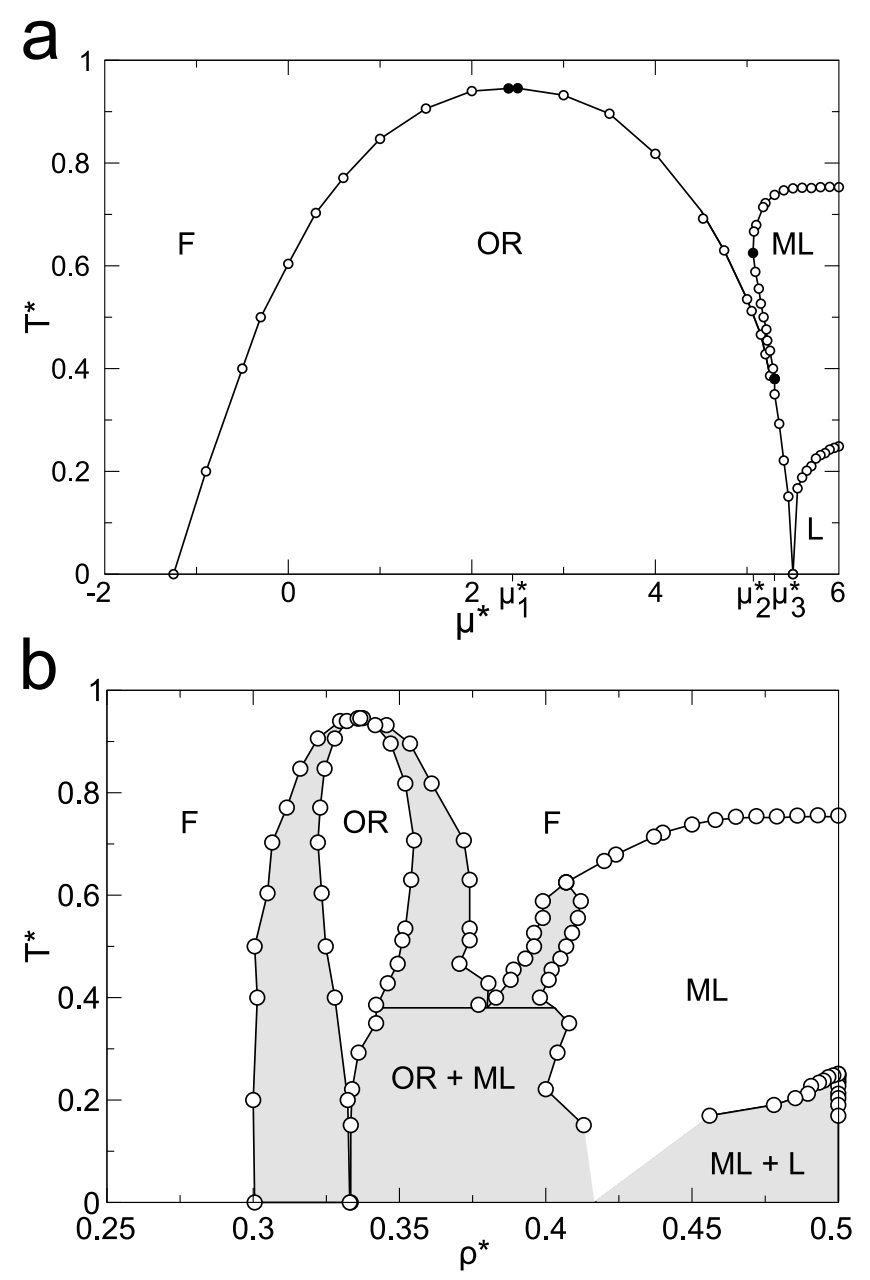

FIG. 3: Phase diagram in the temperature-chemical potential (a) and in the temperature-density (b) planes. OR stands for ordered-rhombus phase, F (fluid) for the disordered phase(s), L represents ordered lamellas, and ML molten lamellas.

rhomboidal clusters. In order to analyze the possible phase transition of the system we have to pay attention to the concavity/convexity properties of the entropy as a function of the density of these clusters (See Refs. [42, 43] for similar situations, in which a low density phase can appear due to the presence of repulsive interactions between particles). In short, we can simulate an athermal lattice gas model where the elementary units are four-particle clusters, with exclusion rules compatible with the lack of repulsive interactions between clusters in the lattice version (LSALR) of the SALR model.

This hard-core lattice gas model allowed us to compute the phase equilibria at conditions close to those where our standard MC algorithm is inefficient, and it provides a consistency check of the simulation codes by comparing its results with those of the LSALR model at 
low temperature.

The possible positions of a rhomboidal cluster on the triangular lattice with $M=L^{2}$ sites can be described by considering the bond connecting the two sites of the rhombus that have three particles in the nearest neighbor $(\mathrm{NN})$ positions. These bond positions define a Kagomé lattice [44] with $3 M$ sites: Each rhombus of the LSALR model on the triangular lattice is mapped on a site of the Kagomé lattice. Taking into account the interactions of the LSALR model it is easy to determine the exclusion rules on the new lattice, i.e. which sites cannot be occupied due to the presence of a particle (occupied site).

The simulation of the hard-core lattice model is performed using two types of moves: translations and changes of the number of particles. Translations are carried out by (1) Selection of a particle at random, (2) Deleting it from the system and updating the list of non-excluded positions, and (3) Inserting it back in one of the allowed positions (chosen at random with equal probabilities). The second type of moves involves changes in the number of particles. This is performed by choosing at random with equal probabilities either an insertion or a deletion attempt: If insertion is chosen and there are allowed positions, one of these allowed positions is selected at random, and acceptance rules are applied. If there are no allowed positions the insertion attempt is directly rejected. Deletion attempts are done by choosing at random a particle in the system to be removed.

Considering detailed balance [38] the ratio between the acceptance probabilities, $A(N \rightarrow$ $N^{\prime}$ ) of an insertion and its reversal deletion move must fulfil

$$
\frac{A(N \rightarrow N+1)}{A(N+1 \rightarrow N)}=\frac{(N+1) z}{N_{\text {pos }}(N)}
$$

where $z$ is the fugacity, and $N_{\text {pos }}(N)$ is the number of non-excluded positions in the configuration with $N$ particles. Using this simulation procedure for $L=120$, coupled with parallel tempering and thermodynamic integration, we obtain the densities of the two phases at coexistence: $\rho_{F} \simeq 0.30$, and $\rho_{O R} \simeq 0.3330$. This result is consistent with the estimate $\rho>0.2$ for the density in the CF phase at the coexistence with the OR phase obtained in Ref. [30] beyond MF.

Interesting features appear at the plots of the density of the fluid phase as a function of $z$. For values of $z$ close to the transition, but still in the region where the fluid phase is the stable one, the quantity: $(\partial \rho / \partial(\ln z))$ shows an oscillatory behavior. In the plot of $\rho$ vs. $\ln z$ (Fig. 4) signatures of apparent weak transitions (steps-like changes in the density) can 


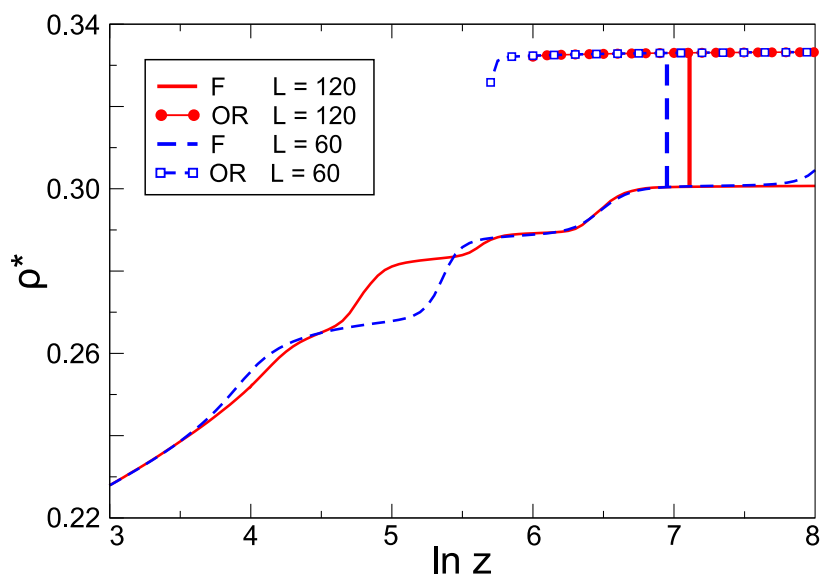

FIG. 4: Density as a function of the logarithm of the fugacity for the hard-core lattice gas model representing the F-OR equilibrium for $T \rightarrow 0$. Continuous line represents the results for the fluid phase and $L=120$. Dashed lines are used for the fluid phase and $L=60$. Lines with symbols mark the results for the OR phase (filled circles for $L=120$, and squares for $L=60$ ). Thick vertical lines mark the estimates for the F-OR transition $(\ln z \simeq 7)$.

be seen. The number of these steps seems to increase with the system size, which implies that the results for the density of the fluid phase close to the transition depend on $L$. We cannot provide a definitive explanation of this behavior. It might occur that in the fluid phase, close to the transition, the system exhibits a short-range order, with small domains of rhombi arranged according to the OR structure. The size of these domains is expected to increase with $z$, the growth of the domains might be conditioned by the effects of the PBC, which impose some correlations which might be relevant as the correlation length of the short-range order increases. Moreover, the use of a lattice model implies additional spatial and orientational correlations which might amplify the effects described above. Within this scenario we could expect that the lines $\rho$ vs $\ln z$ will become smoother for larger system sizes.

\section{F-OR transitions at finite temperature}

Sequences of simulations starting from conditions where the OR phase is expected to be stable: $T \rightarrow 0 ;-1.25<\mu^{*}<5.50$; showed that this ordered phase melts irreversibly (for large system sizes, e.g. $L=120$ ) upon increasing $T^{*}$ to produce phases without long- 
range translational order. We should stress here that no evidence has been found from the simulation results to describe the $\mathrm{CF}$ and the dilute gas as thermodynamically distinct phases. For this reason $\mathrm{F}$, instead of $\mathrm{CF}$, will be used to denote disordered phase(s) at finite temperature.

In order to determine the loci of these melting transitions we firstly assumed that for values of $-1.25<\mu^{*} \leq 5.50$ there are two possible phases, the OR consisting of the ordered rhombuses (stable at low temperature), and a fluid (F) phase. Simulation results suggested that the above assumption was appropriate provided that $\mu^{*}<\mu_{2}^{*} \simeq 5.07$. For values in the range $\mu^{*} \in\left[\mu_{2}^{*}, 5.5\right]$ both, the inspection of system configurations and the use of appropriate order parameters indicates that after the melting of the OR phase, at some temperature range, the system can adopt structures with some lamella character that exhibit orientational order. We will deal with these cases later in the paper.

The loci of the OR-F transition were computed following the methodology explained in Sec. III A in combination with the parallel tempering technique. The results are shown in Fig. 3. For $\mu^{*}<\mu_{1}^{*} \simeq 2.4$ the $\mathrm{F}$ phase is less dense than the OR phase. As one increases the chemical potential from $\mu^{*}=-1.25$, the temperature at the phase equilibrium increases and for temperatures $T^{*}>0.5$ the density of the fluid branch also increases. At $\mu^{*}=\mu_{1}^{*}$ the coexistence line reaches a maximum in temperature at $T_{2}^{*} \simeq 0.95$. At these conditions both phases have the same density $\rho \simeq 1 / 3$, but the OR-F phase transition is still first-order, with a discontinuity in the energy [42, 43]. For $\mu^{*}>\mu_{1}^{*}$ the density is larger in the $\mathrm{F}$ phase at F-OR coexistence.

We found that the grand canonical energy $H$, and the density $\check{\rho}$, for the F phase as a function of the corresponding integration variable showed anomalies similar to those previously discussed for the density of the model for $T \rightarrow 0$, when approaching the F-OR transition.

The $\mathrm{F}$ phase(s) show different types of structures depending on the values of $\mu^{*}$, and $T$. At low temperature, $T^{*} \lesssim 0.5$, and low values of $\mu^{*}$ the system can be described as a gas formed basically by four-particle clusters (rhombuses, See Fig. 1f), resembling the CF phase found for $\mu^{*}=-5 / 4$ at $T=0$. These clusters are favored by energy considerations (sec. II B) and they will be predominant for not too low densities. At higher temperature and density this phase appears as a mixture of clusters of different sizes, which eventually percolate as the chemical potential approaches $\mu^{*}=6$ [44]. Typical snapshots of the high- $T$ OR and $\mathrm{F}$ phases for $\mu^{*}=1$ are shown in Fig.5. 
(a) $\mathrm{T}^{*}=0.833$

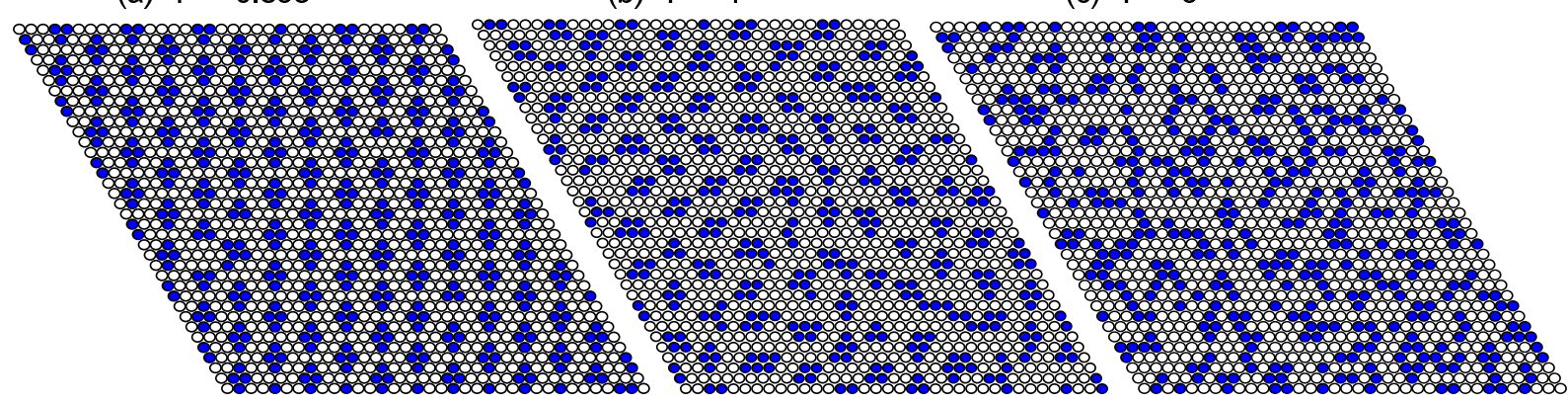

FIG. 5: Representative configurations for $\mu^{*}=1$ with $T^{*}=0.833(\mathrm{OR})$ and $T^{*}=1,3$ (fluid).

\section{Order parameters for lamellar phases}

In this section we introduce two order parameters with the aim of characterizing the presence of lamellas in the medium. In order to do this we will take into account the shape of small fragments of the lamellas in the GS.

\section{Translational order parameter}

Translational order can be checked for a given set of simulation configurations, using the following procedure for each of the three main directions of the lattice: For each row $r=0,1, \ldots, L-1$ in the selected direction $\mathbf{e}_{\alpha}$, where $\alpha=1,2$ or 3 , we consider the states of its $j$ sites: $\hat{\rho}(r, j) \equiv \hat{\rho}\left(j \hat{\mathbf{e}}_{\alpha}+r \hat{\mathbf{e}}_{\beta}\right)$ for $j=0,1,2, \cdots, L-1$ and $\beta \neq \alpha$. The four-site periodicity in the row $r$ in direction $\alpha$ can be checked by computing the parameters $p_{r \alpha}(k)$

$$
p_{r \alpha}(k)=\frac{1}{L}\left|\sum_{j=0}^{L-1}\left\{[2 \hat{\rho}(r, j)-1] g_{k}(j)\right\}\right|,
$$

where $g_{k}(i)$ takes the form of a square wave (Fig, 6) with the values

$$
g_{k}(i)=\left\{\begin{array}{lll}
1 & ; \text { if } & \bmod (i+k, 4)<2 \\
-1 & ; \text { if } & \bmod (i+k, 4) \geq 2
\end{array}\right.
$$

where $k=0$ or 1 and mod is the Modulo operation. A global translational order parameter can be defined for the selected direction, say $\mathbf{e}_{\alpha}$, by combining the results for $k=0$ and $k=1$ :

$$
P_{\alpha}=\frac{1}{L} \sum_{r=0}^{L-1} \max \left[p_{r \alpha}(0), p_{r \alpha}(1)\right]
$$




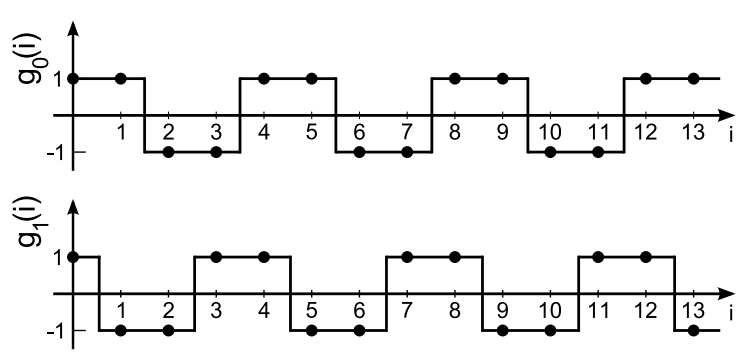

FIG. 6: The function $g_{k}(i)$ (see Eq 11) ) for $k=0$ (top) and $k=1$ (bottom). The lines connecting values of the function for $i=0,1,2, \ldots$ are to guide the eyes.

Lamellar structures with long-range translational order can be identified as those structures for which the order parameter $\mathrm{P}$ defined as

$$
P \equiv \max \left[P_{1}, P_{2}, P_{3}\right]
$$

is close to 1 .

\section{Lamellar stripes and orientational order parameter}

For chemical potentials around $\mu^{*}=6$, the stable configurations at low temperature can be described as ordered lamellar phases, which exhibit long-range translational and orientational order: Lamellar stripes grow only in two out of three main directions of the underlying lattice (see Fig,2). In order to analyze how these ordered structures are transformed, and how the orientational order behaves on heating the system, it is useful to define appropriate order parameters.

First, we need some criteria to decide if a group of particles in a given region of the system belongs to a lamellar stripe. Our criteria works as follows: We first look at sets of three sites forming elementary triangles on the lattice. These triangles will be the units that we will characterize later as either lamellar or non-lamellar. Only triangles with its three sites occupied, denoted as $\mathrm{T}_{3}$, qualify to be lamellar. Notice that these $\mathrm{T}_{3}$ triangles appear also in other stable phases of the system (See Fig. 11). Given a $T_{3}$ triangle, we look at the structure of its neighbor triangles. Suppose that we pay attention to the triangle labelled as 0 in the local structures plotted in Fig. 7. The triangle 0 can be considered as a possible lamellar triangle if precisely two of its NN triangles (labelled as 1 in the figure) have their three sites occupied. Notice that this second criterion excludes all the $\mathrm{T}_{3}$ triangles in 


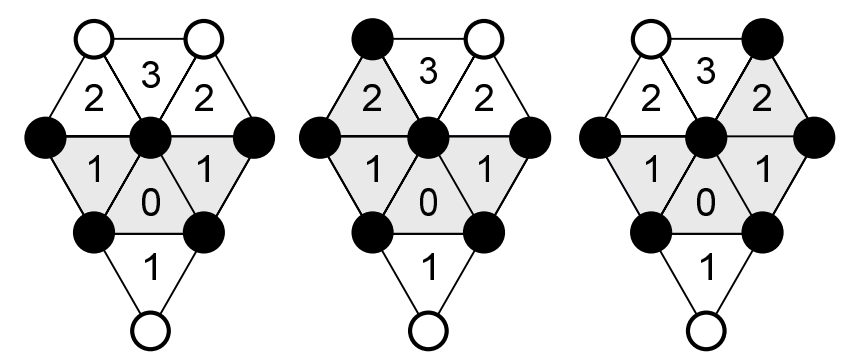

FIG. 7: Sketch of the definition of the lamellar triangle. Filled circles represent occupied sites, empty circles are vacant sites.

the OR structure shown in Fig. 1 $\mathrm{b}$. Finally, in order to exclude from the lamellar type those triangles belonging to possible seven-site hexagonal clusters, we pay attention to the triangles labelled as 2 and 3 in the figure. We consider that 0 is a lamellar triangle if, in addition to the fulfillment of the previous conditions, the triangle labelled as 3 is not fully occupied. According to these rules, the triangle 0 in Fig. 7 is considered as lamellar in the three local structures shown therein. In addition to classifying the fully occupied triangles as lamellar or non-lamellar ones, this method also gives the orientation of the lamellar stripe at that point, just by considering which two out of the three nearest-neighbor triangles are fully occupied. There are three possible orientations which coincide with the principal directions on the triangular lattice.

Visual inspection of different realizations of simulations in the neighborhood of $\mu^{*}=6$ suggests that after the melting of the ordered lamellar phase (the molten phase looses the translational order in the melting) lamella-like stripes which show some orientational order, can still be observed (Fig:8). In order to quantify this behavior and relate it, eventually, with some phase transitions we define appropriate order parameters.

Taking into account the ground state structures for the lamellar phases, and the ability of the lamellar stripes to bend (See Fig. 2 and 8 ) we compute separately the number of lamellar triangles that present one of the three possible orientations: $N_{L T}^{(1)}, N_{L T}^{(2)}$, and $N_{L T}^{(3)}$. If the orientational order exists, two of the orientations will be preferred by the lamellar triangles. Accordingly, we have defined the orientational order parameter for lamellas as:

$$
O_{L}=\frac{N_{L T}-3\left[\min \left(N_{L T}^{(1)}, N_{L T}^{(2)}, N_{L T}^{(3)}\right)\right]}{N_{L T}},
$$

where $N_{L T}=\sum_{i=1}^{3} N_{L T}^{(i)}$ is the total number of lamellar triangles. If no lamellar orientational 


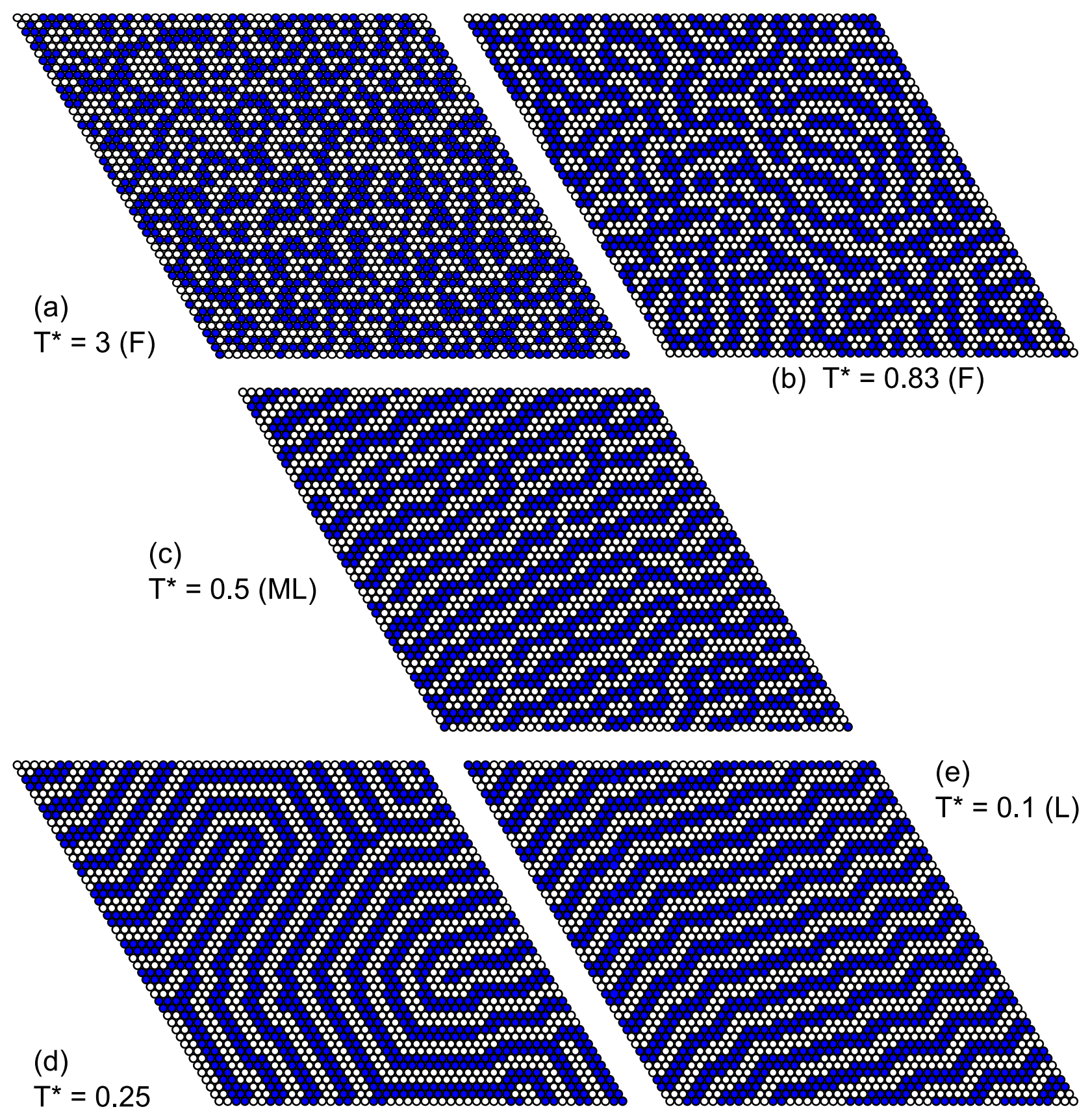

FIG. 8: Representative configurations for the lattice SALR model at $\mu^{*}=6(\langle\rho\rangle=1 / 2)$. The system size is $L=48$.

order exists, then $O_{L}$ will be close to zero, whereas it will approach $O_{L}=1$ if the lamellar stripes exhibit preferential orientations. 


\section{Phase transitions of lamellas}

Parallel tempering was applied for a set of states with constant value of $\mu^{*}$, starting at $\beta=0$ and finishing at low temperature, typically $\beta^{*}=6.0$, with the values of $\beta$ given as $\beta_{i}^{*}=\beta_{0}+i \Delta \beta ;($ with $i=0,1,2, \ldots)$ and $\Delta \beta=0.01$ or $\Delta \beta=0.02$. Several values of $\mu^{*}$ were considered in the range $5.5<\mu^{*} \leq 6$. The initial configurations used for all the replicas were those corresponding to the limit of infinite temperature and finite $\mu^{*}$ (i.e. Each site of the lattice is occupied at random with probability $1 / 2$ ).

For the ordered lamellar phase at low temperature we run sequences of simulations starting at low temperatures, typically $\beta_{0}^{*}=5.0$, using as the starting configuration one of the GS zig-zag lamellas, with subsequent heating steps. We found that the structure of the ordered lamellar phase hardly deviates from the GS, and therefore one can consider directly the ideal structure of this phase and its entropy to compute $\Omega(L, \beta, \mu)$. At some temperature the ordered lamella melts irreversibly (above the equilibrium melting temperature) to produce phases lacking the translational order, but keeping the orientational order. In Fig. 9 we show some results for the case of $\mu^{*}=6$ and $L=120$. By means of TI we found that the melting temperature for the ordered lamellas is relatively low: $T^{*} \approx 0.24$ for $L=120$ and $\mu^{*}=6$. This melting temperature decreases for $\mu^{*}<6$ (See Fig. 3).

Simulation results indicate that there is a range of temperature where the configurations of the system can be described as molten lamellas with orientational order (See Fig. 9). Looking at the variation of the order parameter $O_{L}$ with temperature a sharp transition can be observed. The temperature where this transition occurs depends on $\mu$, and on the system size $L$. At this transition the heat capacity $c_{\mu} \equiv L^{-2}(\partial H / \partial T)_{L, \mu}$, presents a clear peak. The position and the height of this peak have non-monotonic dependences with $L$ (in the range $24 \leq L \leq 120$ ), but the transition is observed for all the system sizes. In spite of the non-monotonic dependence of the local maximum value of the heat capacity $c_{\mu}^{\max }(\mathrm{L})$ with $L$,

the value of $c_{\mu}^{\max }$ seems to increase slightly with $L$ (See Fig[10). These results suggest that the transition is continuous in the range $5.5<\mu^{*} \leq 6.0$. A weakly first-order transition, however, cannot be ruled out.

For temperatures below this order-disorder transition, a number of apparent weak transitions was found. As observed for the F phase close to the F-OR transition, the number of these transitions increases with the system size, whereas the individual jumps in $H / M$ 


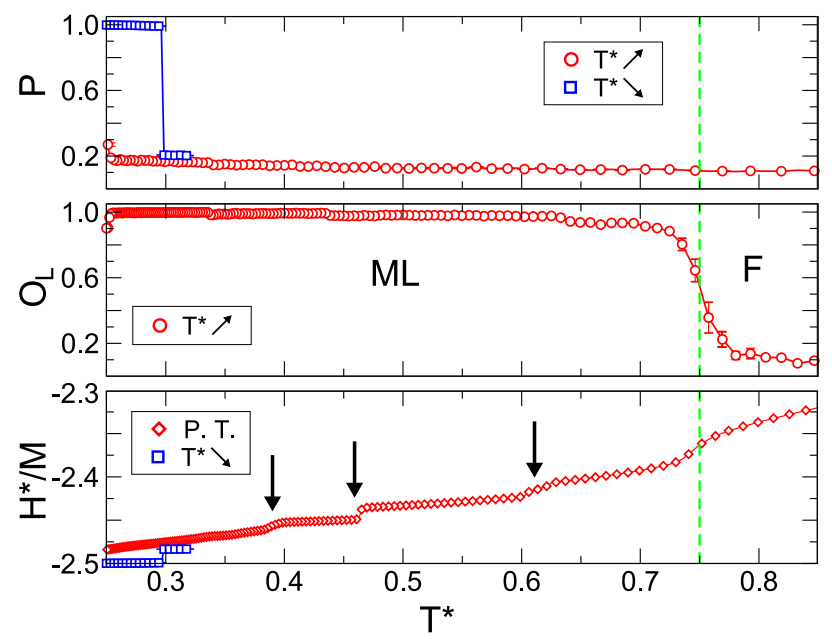

FIG. 9: Simulation results for the analysis of phase transitions in the lamella region. $\mu^{*}=6$, $L=120$. In the top panel we plot the translational order parameter, $P$ (See Eq. (13)), as a function of the temperature: Circles mark the results for a sequence of simulations starting at high temperature, squares represent the results when starting at low $T^{*}$ with the initial configuration corresponding to one of the GS lamellar microstates. Only in the latter case the system exhibits translational order, which is lost when the ordered lamella melts at $T^{*} \simeq 0.3$. In the central panel the orientational order parameter for lamellas, $O_{L}$ (See Eq. (14)) is plotted for a sequence of simulations starting at high temperature: the order-disorder transition at $T^{*} \simeq 0.75$, marked with the dashed vertical lines, can be clearly appreciated. Finally, in the lower panel the Grand Canonical Energy $H^{*}$ per site is plotted: Diamonds mark the results obtained using the Parallel tempering scheme starting from disordered configurations, whereas squares represent the results for the sequence of simulations starting from the ground state configuration. The results from Parallel tempering exhibit signatures of a likely continuous transition at the temperature where the $O_{L}$ indicated an order-disorder transition. In addition some step-like transitions (denoted by arrows) are observed below that temperature.

and $\rho$ (for $\left.\mu^{*}<6\right)$ decrease with $L$. Visual inspection of the configurations (Fig 8 ) and of the plots of the order parameters as a function of the temperature (Fig 9) did not provide any clear conclusion about the nature of these apparent transitions. As in the F case this behavior could be due to effects of compatibility of the stable patterns with the PBC, and the restrictions introduced by considering a lattice model. Note also that the MF stability analysis predicts noninteger period $2 \pi / k_{b}$ of the density oscillations [30], which for the lattice 


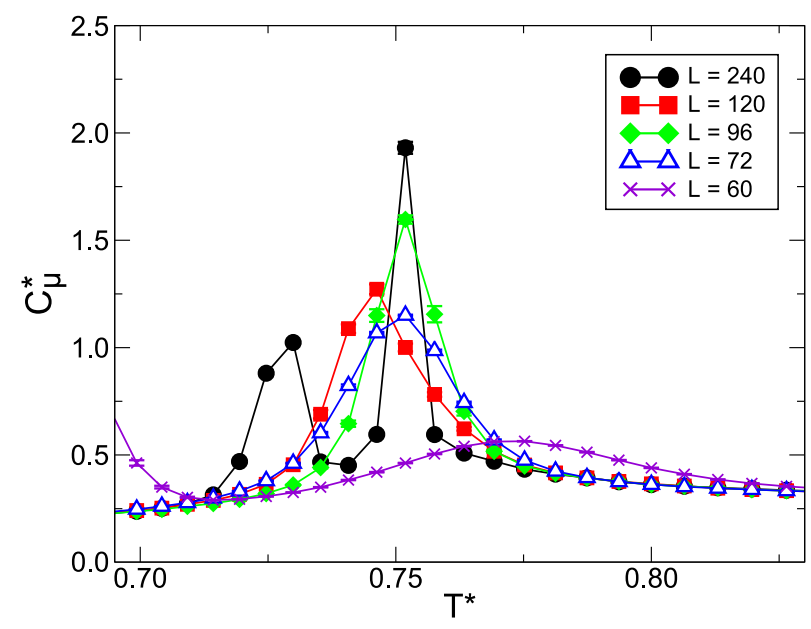

FIG. 10: The heat capacity at the constant chemical potential $c_{\mu}$ for $\mu^{*}=6.0$ and different system sizes; $c_{\mu}$ exhibits a clear peak at the F-ML transition, but also non-monotonic behavior of its position and height for different system sizes.

system together with PBC can lead to strong and nonmonotonic dependence on $L$.

In Fig. 8 we show some representative configurations for the system at $\mu^{*}=6$. In panels a and b configurations of the system in the isotropic $\mathrm{F}$ phase at a high temperature and temperature close to the F-ML transition respectively are shown. In panel b short lamella-like stripes without any preferential orientation, and other cluster structures with local period of 4 in one direction, are present. Fig. 8(c) shows a typical ML structure. Lamellar pieces show preferential orientations. In Fig. 8(d) a metastable configuration for the molten-lamella phase is plotted. The structure of the lamellar stripes is quite regular but the translational order is not achieved and, in addition, the global orientational order is lost. We have found consistently this trend for other system sizes and values of $\mu$ when the temperature is at (or approaching to) the region where the ML phase is metastable with respect to the ordered L phase. Finally, in Fig. 8(e) the ordered lamella structure is presented.

The temperature of both the F-ML and the ML-L phase transitions depends on the chemical potential, and for both transitions assumes the maximum for $\mu^{*}=6$. 


\section{1. $\quad$ The threshold case: $\mu^{*}=5.5$}

In order to understand the system behavior at the threshold value $\mu^{*}=5.5$ we have considered series of simulations using parallel tempering with different system sizes. We used values of $\beta^{*}=1 / T^{*}$ uniformly distributed in the range $0 \leq \beta^{*}<10$. The simulation results showed that for system sizes $L=48,60$, the parallel tempering procedure reaches, at low temperature, configurations with the energy of GS and densities around $\rho=5 / 12$, which correspond to the ML phase shown in Figure. 1 h. For $L=120$ the simulation scheme did not reach these ground state configurations, but the trends of the properties suggest that also in this case the ML will be the stable phase (due to its large entropy) at $\mu^{*}=5.5$. The stability limits of the ML phase at low temperature (say $T^{*}<0.15$ ) in the phase diagrams plotted in Fig. 3 are very hard to compute using our simulation method. Therefore the lines plotted on the $(\rho, T)$ diagram at these conditions have to be seen as conjectural, we cannot exclude the possibility that the ML phase might be stable in a finite range of densities in the limit $T \rightarrow 0$.

\section{Intermediate region $\mu_{2}^{*} \leq \mu^{*}<5.5$}

A more complex behavior is found when analyzing the phase behavior as a function of the temperature at constant chemical potential in the region $\mu_{2}^{*} \leq \mu^{*}<5.5$ (with $\mu_{2}^{*} \simeq 5.07$ for $L=120$ ). In spite of the fact that the GS for this range of chemical potential is the OR phase, on cooling the system at constant $\mu$ one finds that at some temperature $T_{F-M L}(\mu, L)$ there is a $\mathrm{F} \rightarrow \mathrm{ML}$ transition. Depending on the value of $\mu$ this ML phase can eventually melt again at a lower temperature, and the isotropic phase is recovered. Similar reentrant melting of the periodic phase was found in MF in the one-dimensional version of the present model [35]. This reentrant melting appears for $\mu_{2}^{*} \leq \mu \leq \mu_{3}^{*} \simeq 5.30$, and the transition seems to be discontinuous, with a finite change both in the Grand Canonical energy per particle: $H / M$, and in density. Further cooling of the system transforms the fluid into the OR phase

in a discontinuous transition located through TI calculations. In the range $\mu_{3}^{*} \lesssim \mu^{*}<5.5$, no reentrant melting occurs and a discontinuous transition between the ML and OR phases occurs at low temperature.

The reentrant behavior can be observed in the $(\mu, T)$ representation of the phase diagram 
shown in Fig 3. The results for $L=120$ suggest that the transition changes from a continuous to a weakly first order at a temperature $T^{*} \simeq 0.6($ Fig $3 \mathrm{~b})$, and that this change occurs at, or close to, $\mu_{2}^{*}$, i.e. the minimum value of the chemical potential with the F-ML equilibrium.

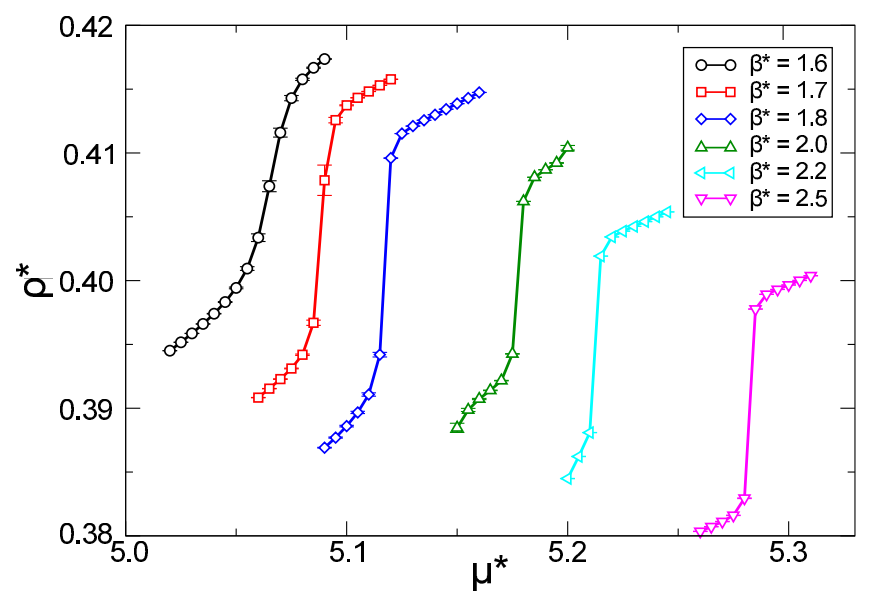

FIG. 11: Density as a function of the chemical potential for different values of $\beta^{*}$ at the $\mathrm{F} \rightarrow \mathrm{ML}$ transition.

The calculation of the F-ML transition below $T^{*} \simeq 0.6$ was carried out by fixing the temperature and running parallel tempering $\mathrm{MC}$ simulation using several values of the chemical potential. The plots of the density as a function of the chemical potential indicated a continuous transition for $\beta^{*}=1.60$, whereas for $\beta^{*} \geq 1.70$, a clear discontinuity was observed (Fig.11). From these plots it was possible to get estimates for both, the chemical potential at the transition, and the density of the two phases.

The topology of the phase diagram implies the existence of a triple point where the OR, F, and ML phases coexist. Using the results of simulations with $L=120$ we have estimated the locus of this point as: $\mu_{T P}^{*}=\mu_{3}^{*} \simeq 5.3, T_{T P}^{*} \simeq 0.38$.

\section{DISCUSSION AND SUMMARY}

We have used MC simulation to compute the phase diagram of a triangular lattice model with the SALR interactions. We focused on the case of strong repulsion, with the interaction potential $V(\mathbf{x})$ such that $\sum_{\mathbf{x}} V(\mathbf{x})>0$. The main features of the phase diagram in our model and in the case of the SALR potential with moderate repulsion in an off-lattice system [14, 15] are similar. The details, however, are significantly different. The most important novelty is 
the stability of two different lamellar phases.

Let us compare the MF and the MC phase diagrams for the repulsion to attraction ratio $J^{*}=3$. In the $\left(\mu^{*}, T^{*}\right)$ variables the phase diagrams (Fig. 6 in Ref.[30] and Fig.33) are similar for low $T^{*}$, except that in $\mathrm{MC}$ the stability region of the ML phase extends down to $T^{*}=0$, while in $\mathrm{MF}$ the $L_{2}$ phase appears for $T^{*}>0.65$. The relation between the ML and the $\mathrm{L}_{2}$ phases obtained in MC and in MF respectively is not entirely obvious. In the $L_{2}$ phase the density oscillates in one of the lattice directions $\mathbf{e}_{i}$, i.e the distinguished directions in the $L_{2}$ and the ML phases are the same. The structure of the ML phase resembles the structure of the L phase with defects. The defects do not destroy the orientational order, but the translational order is lost (see Fig,8c). We attribute the stability of the ML phase for $T^{*} \rightarrow 0$ to the effect of the degeneracy of the GS that cannot be properly described within MF.

For high $T^{*}$ the differences between the MF and MC phase diagrams in the $\left(\mu^{*}, T^{*}\right)$ variables are more pronounced. In MF the stability region of the ordered phases extends to much higher temperatures than found by $\mathrm{MC}$ simulation. Moreover, for high $T^{*}$ the $\mathrm{H}$ (hexagonal) phase with translational and without orientational order of the rhomboidal clusters appears in the MF, but not in the MC phase diagram. Although we were not able to identify the $\mathrm{H}$ phase using MC simulation, we cannot rule out the physical significance of this MF result. Notice that in our analysis of the simulation results (see for instance Fig. 4) we found some anomalous behavior both in the equations of state (either $\rho(T)$ at constant $\mu$, or $\rho(\mu)$ at constant $T$ ), and in the dependence of the results on the system size in the putative region of the $\mathrm{F}$ phase close to the transition to the OR phase. In this regard, it is interesting to point out that other models on the triangular lattice and related models containing competing interactions [45-48] exhibit modulated structures, and the phenomenology of Devil's staircase [49]. Our simulation analysis cannot answer if it is the case for our lattice SALR model, but both the behavior of the fluid phase close to the transition to the OR phase, and the results in the region described as molten lamellas exhibit some similarities with those reported for this kind of systems.

Further differences between the phase diagrams concern the sequences of phase transitions for high $T^{*}$. In MF the $L_{2}$ phase coexists with the $\mathrm{H}$ phase for high $T^{*}$, and extends to higher $T^{*}$ than the $\mathrm{OR}$ phase. In contrast, in MC we find that the ML phase coexists with the disordered $\mathrm{F}$ phase for high $T^{*}$, and extends to lower $T^{*}$ than the OR phase. We attribute 
the stability of the F phase between the OR and ML phases to the effects of fluctuations.

In our $\mathrm{MC}$ simulation we do not discriminate between possibly distinct fluid $\mathrm{F}$ phases, despite signatures of the phase transitions both in the density and in the energy. Thus, the region marked as $\mathrm{F}$ in Fig.3 might in fact represent stability of a few different phases (see the snapshots in Fig.8).

The differences between the MF and MC phase diagrams are even stronger in the $\left(\rho, T^{*}\right)$ variables. The density ranges of the stability regions of the ordered phases and the twophase regions are quite different in $\mathrm{MF}$ and in $\mathrm{MC}$. The exception is the ordered $\mathrm{L}$ phase. It is stable for very narrow range of densities, $\rho \approx 1 / 2$ both in MF and in MC.

On the MC phase diagram the ML phase coexists with the $\mathrm{F}$ phase for $T^{*}$ higher than the temperature at the triple point OR-F-ML, and the transition between the two phases becomes continuous or very weakly first-order above $T^{*} \simeq 0.6$. In MF we found a continuous phase transition between the $\mathrm{F}$ and the $L_{2}$ phases only for $\rho=1 / 2$ [30]. We have calculated the maximum $S_{m}$ of the structure factor $S(k)$ in the $\mathrm{F}$ phase in the Brazovskii-type approximation [31]. We have obtained a large value, $S_{m} \simeq 10^{4}$ for $T^{*}=0.8$. For decreasing $T^{*}$ the maximum of the structure factor increases, but it diverges only for $T^{*}=0$. This means a very large but finite correlation length, and an absence of the continuous transition to the phase with periodic density for $T^{*}>0$ beyond MF. Recently, however, a continuous transition between the isotropic and the nematic phases has been predicted for a stripeforming system in the Brazovskii $\varphi^{4}$ theory at two-loop order[32]. This theory is relevant for $\rho=1 / 2$ in our model, and supports the MC results for the transition between the disordered and the ML phases. We expect that for high $T^{*}$ the transition F-ML is continuous, but since we do not have a definite proof for the order of the transition, the very weakly first-order transition is not ruled out.

Based on the comparison of the $\mathrm{MF}$ and $\mathrm{MC}$ phase diagrams we conclude that if the amplitude of the density wave in an ordered phase is small in MF, then only a shortrange order (such as in Fig 8b) is left in a presence of fluctuations. The MF ordered phases with a large amplitude of the density oscillations remain stable in a presence of fluctuations. However, instead of a coexistence of two different ordered phases, we find a transition between each of them and a less ordered phase above a triple point. We found the OR-ML-L triple point at $T^{*}=0$, and the OR-F-ML triple point at $T^{*} \simeq 0.38$. We may observe that the temperature of the triple point is higher when the coexisting phases are 
less ordered.

A further interesting question is: to what extent the results for the lattice SALR model can be extrapolated to the corresponding models in the continuum? Regarding this point we could expect that the existence of low density ordered phases analogous to our OR phase might strongly depend on the details of the interaction potential. In the continuum, a low density cluster ordered phase could be expected only if the clusters formed at low temperature show little amount of polydispersity both in a number of particles and shape. On the other hand, the transitions involving isotropic phases and lamellar structures seem to appear easily for systems in the continuum [13-15, 19, 50]. We think that the results presented in this work will provide a convenient framework to analyze the possible transitions between fluid and lamellar phases, and the possible order-disorder transition between different lamellar phases.

Finally, we should mention that experimental observation of thermodynamically stable patterns is a real challenge, and our results can guide the experimental studies.

\section{ACKNOWLEDGMENTS}

The work of JP was realized within the International PhD Projects Programme of the Foundation for Polish Science, cofinanced from European Regional Development Fund within Innovative Economy Operational Programme "Grants for innovation". NGA gratefully acknowledges financial support from the Dirección General de Investigación Científica y Técnica under Grant No. FIS2010-15502, from the Dirección General de Universidades e Investigación de la Comunidad de Madrid under Grant No. S2009/ESP-1691 and Program MODELICO-CM. We also acknowledge the financial support by the NCN grant 2012/05/B/ST3/03302 
[1] M. Seul and V. S. Chen, Phys. Rev. Lett. 70, 1658 (1993).

[2] M. Seul and D. Andelman, Science 267, 476 (1995).

[3] C. S. Scheve, P. A. Gonzales, N. Momin, and J. C. Stachowiak, J. Am. Chem. Soc 135, 1185 (2013).

[4] Q. Fan, C. Wang, Y. Han, J. Zhu, J. Kuttner, G. Hilt, and J. M. Gottfried, ACS Nano (2013), doi: $10.1021 / \mathrm{nn} 405370$ s.

[5] S. Reynaert, P. Moldenaers, and J. Vermant, PCCP 9, 6463 (2007).

[6] P.-F. Lenne and A. Nicolas, Soft Matter 5, 2841 (2009), doi: 10.1039/b822956b.

[7] T. Lang and S. O. Rizzoli, Physiology 25, 116 (2010), doi: 10.1152/physiol.00044.2009.

[8] R. El-Khouri, S. L. Frey, A. W. Szmodis, E. Hall, K. J. Kauffman, T. E. P. andKa Yee C. Lee, and A. N. Parikh, Langmuir 27, 1900 (2011).

[9] C. Stefaniu, G. Brezesinski, and H. Möhwald, Soft Matter 8, 7952 (2012).

[10] C. S. Thomas, L. Xu, and B. D. Olsen, Biomacromolecules 13, 2781 (2012), doi: 10.1021/bm300763x.

[11] R. P. Sear and W. M. Gelbart, J. Chem. Phys. 110, 4582 (1999).

[12] D. Pini, G. Jialin, A. Parola, and L. Reatto, Chem. Phys. Lett. 327, 209 (2000).

[13] A. Imperio and L. Reatto, J. Phys.:Cond. Mat. 18, S2319 (2004).

[14] A. Imperio and L. Reatto, Phys. Rev. E 76, 040402 (2007).

[15] A. Imperio and L. Reatto, J. Chem. Phys. 124, 164712 (2006).

[16] D. Pini, A. Parola, and L. Reatto, J. Phys.:Cond. Mat. 18, S2305 (2006).

[17] A. J. Archer, D. Pini, R. Evans, and L. Reatto, J. Chem. Phys. 126, 014104 (2007).

[18] A. Ciach, Phys. Rev. E 78, 061505 (2008).

[19] A. J. Archer, Phys. Rev. E 78, 031402 (2008).

[20] J.-M. Bomont and D. Costa, J. Chem. Phys. 137, 164901 (2012), doi: 10.1063/1.4759503.

[21] F. Bischoff, K. Seufert, W. Auwarter, S. Joshi, S. Vijayaraghavan, D. Ecija, K. Diller, A. C. Papageorgiou, S. Fischer, F. Allegretti, et al., ACS Nano 7, 3139 (2013).

[22] J. N. Israelachvili, Intermolecular and Surface Forces (Third Edition) (Academic Press, Boston, 2011).

[23] A. Shukla, E. Mylonas, E. D. Cola, S. Finet, P. Timmins, T. Narayanan, and D. I. Sveergun, 
Proc. Nat. Acad. Sci. USA 105, 5075 (2008).

[24] A. Stradner, H. Sedgwick, F. Cardinaux, W. Poon, S. Egelhaaf, and P. Schurtenberger, Nature 432, 492 (2004).

[25] A. I. Campbell, V. J.Anderson, J. S. van Duijneveldt, and P. Bartlett, Phys. Rev. Lett. 94, 208301 (2005).

[26] A. Sanchez-Iglesias, M. Grzelczak, T. Altantzis, B. Goris, J. Perez-Juste, S. Bals, G. V. Tondeloo, S. H. Donaldson, B. F. Chmelka, J. N. Israelachvili, et al., ACS Nano 6, 11059 (2012).

[27] T. Lafitte, S. Kumar, and A. Z. Panagiotopolous, Soft Matter 70, 786 (2014), doi: 10.1039/C3SM52328D.

[28] M. Dijkstra, R. van Roij, and R. Evans, Phys. Rev. E 59, 5744 (1999).

[29] P. Kowalczyk, A. Ciach, P. A. Gauden, and A. P. Terzyk, Int. J. Colloid and Interf. Sci. 363, 579 (2011).

[30] J. Pȩkalski, A. Ciach., and N. G. Almarza (2014), arXiv:1401.0801 [cond-mat.soft].

[31] S. A. Brazovskii, Sov. Phys. JETP 41, 8 (1975).

[32] D. G. Barci, A. Mendoza-Coto, and D. A. Stariolo, Phys. Rev. E 88, 062140 (2013).

[33] A. de Candia, E. DelGado, A. Fierro, N. Sator, M. Tarzia, and A. Coniglio, Phys. Rev. E 74, 010403(R) (2006).

[34] A. B. de Oliveira and M. C. Barbosa, J. Phys.:Cond. Mat. 17, 399 (2005), doi: 10.1088/0953$8984 / 17 / 3 / 001$.

[35] J. Pȩkalski, A. Ciach, and N. G. Almarza, J. Chem. Phys. 138, 144903 (2013).

[36] A. Ciach, J. Mol. Liquids 164, 74 (2011).

[37] M. P. Allen and D. J. Tildesley, Computer Simulation of Liquids (Oxford University Press, 1987).

[38] D. Frenkel and B. Smit, Understanding Molecular Simulation. From Algorithms to Applications (Academic Press, Boston, 1996).

[39] N. G. Almarza, J. M. Tavares, E. G. Noya, and M. M. T. da Gama, J. Chem. Phys. 137, $244902(2012)$.

[40] R. H. Swendsen and J.-S. Wang, Phys. Rev. Lett. 57, 2607 (1986), URL http://link.aps.org/doi/10.1103/PhysRevLett.57.2607.

[41] D. J. Earl and M. W. Deem, Phys. Chem. Chem. Phys. 7, 3910 (2005), URL 
http://dx.doi.org/10.1039/B509983H.

[42] J. Hoye, E. Lomba, and N. Almarza, Mol. Phys. 107, 321 (2009).

[43] N. G. Almarza, J. A. Capitán, J. A. Cuesta, and E. Lomba, J. Chem. Phys. 131, 124506 (2009).

[44] S. Torquato, Random Heterogeneous Materials, Microstructures and Macroscopic Properties (Springer-Verlag, New York, 2002).

[45] K. Nakanishi, J. Phys. Soc. Jpn. 52, 2449 (1983).

[46] Y. Dublenych, Phys. Rev. E 80, 011123 (2009).

[47] M. E. Fisher and W. Selke, Phys. Rev. Lett. 44, 1502 (1980).

[48] W. Selke and M. E. Fisher, Z. Phys. B 40, 71 (1980).

[49] P. Bak, Rep. Prog. Phys. 45, 587 (1982).

[50] C. J. Olson Reichhardt, C. Reichhardt, and A. R. Bishop, Phys. Rev. E 82, 041502 (2010), 